\title{
METODOLOGÍAS DE APRENDIZAJE ACTIVO EN PROYECTOS ARQUITECTÓNICOS Y SU INCIDENCIA EN LA MOTIVACIÓN DEL ALUMNADO UNIVERSITARIO
}

\section{ACTIVE LEARNING METHODOLOGIES IN ARCHITECTURAL PROJECTS AND ITS IMPACT ON THE MOTIVATION OF UNIVERSITY STUDENTS}

http://dx.doi.org/10.15304/ie.29.5918

\author{
Ricardo Carcelén González \\ Universidad Politécnica de Cartagena \\ ricardo.carcelen@upct.es
}

\section{RESUMEN}

La asignatura de Proyectos en Arquitectura ha permitido desde antaño la aplicación de metodologías de aprendizaje activo basado en proyectos propiciadas por su enseñanza en formato taller y su sistema de evaluación de trabajos y porfolios. Método. El trabajo se marca como objetivo evaluar la incidencia del empleo de este tipo de metodologías sobre la motivación de los alumnos universitarios. Para ello, se analizan diferentes metodologías implementadas durante los tres últimos cursos académicos en la asignatura Proyectos 6 del Grado en Fundamentos de Arquitectura de la Universidad Politécnica de Cartagena. Resultados. En este trabajo se someten a evaluación tres variantes de la metodología de aprendizaje activo basado en proyectos: método activo convencional, aprendizaje activo interdisciplinar y, finalmente, aprendizaje-servicio. Para cada una de ellas se presenta un doble sistema de valoración: cuantitativo, que analiza parámetros como la tasa de abandono, la tasa de éxito o el rango de calificaciones; y cualitativo, evidenciando el grado de satisfacción del alumnado respecto a la implementación de los diferentes instrumentos de innovación educativa, así como las propias metodologías, que aquí se presentan. Conclusiones. El análisis comparado de las diversas variantes de la metodología de aprendizaje activo basado en proyectos nos lleva a presumir una mayor influencia de las metodologías de aprendizaje-servicio sobre la motivación del alumnado universitario, que asumen la utilidad de la tarea a través de la prestación de servicio a la Comunidad como un incentivo complementario que incrementa su implicación con la asignatura objeto de este estudio.

Palabras clave: aprendizaje activo, proyectos arquitectónicos, arquitectura, motivación

\section{ABSTRACT}

The subject of Projects in Architecture has allowed from the past the application of active learning methodologies based on projects promoted by its teaching in workshop format and its evaluation system of works and portfolios. Method. The work is aimed at evaluating the incidence of the use of 
this type of methodologies on the motivation of university students. For this, different methodologies implemented during the last three academic years are analyzed in the subject Projects 6 of the Degree in Fundamentals of Architecture of the Polytechnic University of Cartagena. Results. In this paper, three variants of the active learning methodology based on projects are evaluated: conventional active method, interdisciplinary active learning and, finally, service-learning. For each one of them, a double valuation system is presented: quantitative, which analyzes parameters such as the abandonment rate, the success rate or the rank of qualifications; and qualitative, evidencing the degree of student satisfaction with the implementation of the different educational innovation instruments, as well as the methodologies themselves, which are presented here. Conclusions. The comparative analysis of the different variants of the methodology of active learning based on projects leads us to presume a greater influence of service-learning methodologies on the motivation of university students, who assume the usefulness of the task through the provision of service to the Community as a complementary incentive that increases its involvement with the subject of this study.

Keywords: active learning, architectural projects, architecture, motivation.

\section{INTRODUCCIÓN}

La asignatura de Proyectos en los estudios superiores de Arquitectura (o Fundamentos de Arquitectura si nos ceñimos a la nomenclatura de los Grados actuales) representa el eje vertebrador así como la mayor carga de créditos de los planes de estudios de las diferentes escuelas de Arquitectura en España (y Europa ciertamente); tiene docencia en la totalidad de los niveles del grado; y trata de aunar en una única materia todo el conocimiento adquirido por los estudiantes universitarios en el resto de las asignaturas mediante la aplicación del mismo a un proyecto arquitectónico. El sistema de evaluación imperante es, por tanto, el de valoración de trabajos y porfolios (entregas), por lo que se dan las condiciones idóneas para la puesta en práctica de metodologías de aprendizaje activo o basado en proyectos tal y como se trata de poner de manifiesto en este trabajo.

De hecho, no resulta una novedad el empleo de este tipo de metodologías docentes en la asignatura de Proyectos Arquitectónicos, sino que más bien ha sido el método de enseñanza aplicado tradicionalmente desde que los estudios de Arquitectura tienen una condición reglada. En ese sentido, resultan muy clarificadoras las palabras de Valero (2006, p. 55) cuando señala que:

Un curso de Proyectos es un camino que se recorre explorando un territorio desconocido; el espacio que recorra cada uno será su camino; el guía es el profesor que, por ser más experimentado intentará orientar y marcar el paso, pero se hace camino al andar, como dice el poeta, lo que a nuestros efectos se traduce como aprender a proyectar proyectando.

Una afirmación en total sintonía con las teorías del "aprendizaje experiencial" de Dewey (1916) de principios del siglo pasado —o las más recientes del "aprendizaje auto-regulado", "aprendizaje independiente" o "aprendizaje autónomo" de otros tantos autores señalados por Huber (2008, p. 65) - y que no es difícil de conectar con la estrategia de soporte denominada con el anglicismo coaching del modelo de David H. Jonassen recuperado por García Martín y Pérez Martínez (2018, p. 52) para referirse al acompañamiento y apoyo del tutor académico en el camino del alumnado hacia la resolución del proyecto requerido. 
Sin embargo, y a pesar de la aparente consolidación de estas metodologías de aprendizaje activo en el área de conocimiento Proyectos Arquitectónicos, Santander (2017, p. 163) pone de manifiesto cómo éstas, en términos generales en el contexto universitario, se han estado llevando a cabo por multitud de docentes sin ser siquiera conscientes de su aplicación. En el caso del profesorado en Arquitectura — y de las ingenierías por extensión—, quienes proceden fundamentalmente de una titulación que no incluía formación pedagógica alguna, es habitual que este tipo de metodologías se hayan estado implementando de manera espontánea e intuitiva, tomando partido de la experiencia de los docentes en el desarrollo de proyectos arquitectónicos en el contexto profesional. La única carencia de esas experiencias educativas por satisfacer sería probablemente, como señala de nuevo Santander, "cumplir con el incremento de la reflexión y de sistematizar las experiencias por medio de las evaluaciones".

\section{MÉTODO: EL APRENDIZAJE ACTIVO BASADO EN PROYECTOS}

El objetivo fundamental que se marca este trabajo consiste en determinar el grado de repercusión que sobre la motivación de los estudiantes universitarios de Arquitectura tiene el empleo de las metodologías de aprendizaje activo en las asignaturas de Proyectos Arquitectónicos. Para satisfacer dicho objetivo, se han abordado los últimos tres cursos académicos de la asignatura Proyectos 6 del Grado en Fundamentos de Arquitectura de la Universidad Politécnica de Cartagena con planteamientos metodológicos de aprendizaje activo, cada uno de ellos con variaciones en el enfoque formativo para poder evaluar cuáles de las experiencias educativas ensayadas ha resultado ser más motivadora para el alumnado. La necesidad de explorar métodos de motivación para los estudiantes universitarios surge ante algunas afirmaciones pesimistas de autores como Rinaudo, Chiecher y Donolo (2003, p. 116), quienes señalan que no basta con pensar que "los alumnos asisten a la universidad por voluntad propia, que han elegido una carrera que les gusta y que, en consecuencia, tendrían que estar motivados".

Pese a la existencia de multitud de manuales y guías para el diseño y desarrollo de metodologías de aprendizaje activo como la propuesta de Silberman (1998), en este texto destacamos sin embargo el trabajo de García Martín y Pérez Martínez (2018, p. 43) por su capacidad de concreción y síntesis a la hora de marcar las pautas para el diseño de las actividades a implementar en el método de enseñanza que nos ocupa. Son dichos autores quienes, de hecho, nos introducen en la definición del aprendizaje activo basado en proyectos y nos ayudan a diferenciarlo de aquel basado en problemas. En ese sentido, variables como que se otorgue mayor importancia al resultado final que al proceso o procedimiento para alcanzarlo; la mayor complejidad a la hora de proponer las tareas; la consideración de periodos de trabajo más dilatados en el tiempo; la tendencia a integrar conocimientos previamente adquiridos en la titulación más que a introducir otros nuevos; $\mathrm{y}$, finalmente, la importancia que adquieren cuestiones como la interdisciplinaridad en el diseño de las actividades, son las que nos llevan a considerar las experiencias educativas que aquí se presentan dentro de los planteamientos metodológicos identificables en el aprendizaje activo basado en proyectos, no en problemas.

La asignatura de Proyectos Arquitectónicos representa el marco académico idóneo donde plantear las tareas de tipo ill-structured fundamentales para la correcta implementación de la 
metodología de aprendizaje activo basado en proyectos. Dicho término inglés se utiliza para referirse a aquellas formulaciones en las que no existe una única solución válida al caso de estudio, sino al contrario, múltiples soluciones posibles y diversos caminos y procedimientos para alcanzar alguna de ellas (García Martín y Pérez Martínez, 2018, p. 41-42). Dicho rasgo de identidad es precisamente lo que identifica también al proyecto de arquitectura, donde no existe una única materialización del programa de necesidades válida, sino tantas como procedimientos razonados sea capaz de desarrollar la figura del arquitecto.

En cuanto al diseño de las tareas llevadas a cabo durante los tres últimos cursos académicos, y que nos han permitido llevar a cabo los ensayos necesarios para abordar el estudio que nos ocupa, se ha tenido en cuenta el diseño por fases o etapas habitual en la metodología de aprendizaje basado en proyectos, a saber:

\section{Definición de la actividad}

Las tareas que se han propuesto en cada uno de los cursos académicos objeto de este estudio se ajustan a lo que Graaff y Kolmos (2003) se refieren cuando hablan de problem projects — “a fullscale project in which the course of action is not planned in detail by the teachers" -, en los que se requiere de un alto grado de autonomía de los alumnos implicados en la experiencia educativa, y que en nuestro caso concreto, al encontrarse todos ellos cursando los últimos niveles académicos del Grado, nos permite presuponer la tenencia de las destrezas precisas advertidas por Huber (2008, p. 72).

\section{Soporte durante la actividad}

Tal y como ya se señaló, el tipo de soporte puesto en práctica en dicha experiencia educativa ha sido el de coaching. A la hora de decidir el grado de implicación del profesorado en cada uno de los procedimientos llevados a cabo por los alumnos para la resolución del problema resulta fundamental conocer el grado de conocimiento y formación adquiridos por éstos en el momento de comenzar la actividad. Por ello, y con el fin de asegurar la presuposición referida anteriormente, se requiere a los alumnos la elaboración de un portafolio de trabajos académicos desarrollados en niveles previos que permita evaluar al docente diseñador de las actividades las destrezas de partida de los sujetos participantes y formular la propuesta formativa en consecuencia.

Es en esta fase donde mayor incidencia puede lograrse en términos de motivación del alumnado. Así, al igual que señalan García Martín y Pérez Martínez (2018, p. 51), ha resultado útil la implementación del modelo ARCS — attention, relevance, confidence and satisfaction - que definiese Keller (1987). La atención y la relevancia de las tareas se consiguen con el diseño de unos enunciados de trabajo que propone a los alumnos abordar una tarea académica con planteamientos reales y procedentes de problemáticas actuales, lo que propicia el interés de unos estudiantes que perciben como sus esfuerzos traspasan los límites del ente universitario. La confianza de los alumnos se obtiene tras evidenciar su aptitud y capacidad para superar la tarea propuesta, para lo cual resulta de gran eficacia dar a conocer desde el inicio la rúbrica de evaluación de la tarea, o expresado en otros 
términos, aquello que es requerido al alumno para superar la asignatura. Finalmente, la satisfacción en nuestro caso se canaliza a través de la oferta de incentivos externos, poniendo al alcance de los alumnos la posibilidad de difundir sus trabajos fuera del contexto universitario, bien formando parte de una exposición pública, bien integrando una publicación final de los resultados académicos obtenidos.

\section{Organización de la actividad}

Una vez satisfechas las dos fases anteriores, los docentes deben planificar y organizar cada una de las actividades que estructurarán la experiencia educativa, lo que en nuestro caso nos ha llevado a poner en práctica clases magistrales, el trabajo de campo, el trabajo en equipo, la celebración de talleres colaborativos y participativos, la celebración de jurys, etcétera. Con esta última fase, concluirían las etapas de preparación de la tarea necesarias cuando implementamos metodologías de aprendizaje activo basado en proyectos (García Martín y Pérez Martínez, 2018), y que se han tenido en cuenta para diseñar cada una de las experiencias educativas objeto de este trabajo.

\section{RESULTADOS: APLICACIÓN A PROYECTOS ARQUITECTÓNICOS}

En este trabajo se presentan los resultados obtenidos en tres cursos académicos diferentes y consecutivos de la asignatura de Proyectos Arquitectónicos a la que hacíamos referencia en el punto anterior. En todos ellos se han implementado metodologías de aprendizaje activo basado en proyectos, añadiendo en cada uno de ellos algún parámetro diferenciador que nos permita posteriormente realizar un análisis comparativo y determinar en última instancia qué metodología ha resultado de mayor eficacia en lo que a motivación del alumnado universitario se refiere.

La siguiente tabla (1) resume la metodología empleada, así como el número de alumnos participantes en cada uno de los cursos académicos que han sido objeto de estudio en este trabajo:

\begin{tabular}{|l|l|l|l|}
\hline Curso & Lema del curso & Metodología & Alumnos \\
\hline $2016-2017$ & Paisajes Alterados & Aprendizaje activo & 79 \\
\hline $2017-2018$ & $\begin{array}{l}\text { MUCC: } \\
\text { Cultura para repensar la ciudad }\end{array}$ & Aprendizaje interdisciplinar & 61 \\
\hline $2018-2019$ & Conexiones Vitales & Aprendizaje-servicio & 39 \\
\hline
\end{tabular}

Tabla 1. Metodologías de aprendizaje activo en Proyectos Arquitectónicos. Fuente: elaboración propia.

\section{Aprendizaje activo en Proyectos Arquitectónicos}

Bajo el lema "Paisajes alterados", la asignatura Proyectos 6 del Grado en Fundamentos de Arquitectura planteaba a sus 79 alumnos la participación en una experiencia educativa diseñada para ser desarrollada siguiendo una metodología convencional de aprendizaje activo basado en proyectos, cuyas principales características se han tratado de evidenciar en el apartado anterior. Durante el cuatrimestre, organizado en diferentes fases de trabajo — análisis, estrategias, propuesta 
y resolución-, los alumnos trabajaron de forma completamente autónoma en sus propuestas arquitectónicas con la tutorización — coaching — de los docentes de la asignatura, y con la celebración de un jury final - jurado integrado por agentes especialistas y externos a la asignatura que emiten una valoración objetiva tras una breve presentación oral y pública del trabajo realizado por los propios alumnos (Carcelén, López y Aroca, 2014)—.

\begin{tabular}{|l|l|l|l|l|l|l|l|}
\hline \multicolumn{2}{|l|}{ Curso 2016-2017. Paisajes alterados } \\
\hline NP & Susp. & Ap. & Not. & Sob. & M.H. & Total & \\
\hline 21 & 7 & 36 & 12 & 3 & - & 79 & Tasa de éxito: $87,90 \%$ \\
\hline $\mathbf{2 6 , 5 0 \%}$ & $8,90 \%$ & $45,60 \%$ & $15,20 \%$ & $3,80 \%$ & - & $100 \%$ & Tasa de fracaso: $12,10 \%$ \\
\hline
\end{tabular}

Tabla 2. Resultados cuantitativos aprendizaje activo en Proyectos Arquitectónicos. Fuente: elaboración propia.

\begin{tabular}{|l|l|l|l|l|l|l|l|}
\hline \multicolumn{7}{|l|}{ Valoración global de la metodología de aprendizaje activo basado en proyectos } \\
\hline 1 & 2 & 3 & 4 & 5 & Población & Muestra & Media \\
\hline 0 & 0 & 2 & 6 & 4 & 79 & 12 & $\mathbf{4 , 1 7}$ \\
\hline
\end{tabular}

Tabla 3. Resultados cualitativos aprendizaje activo en Proyectos Arquitectónicos. Fuente: elaboración propia.

Finalizado el cuatrimestre, y en ausencia de parámetros adicionales de innovación educativa a valorar, se evalúa la repercusión sobre la motivación del alumnado de la metodología señalada a través de un estudio cuantitativo de las tasas de abandono y de las tasas de éxito durante el curso académico en cuestión (tabla 2). La valoración anterior se complementa con una segunda evaluación cualitativa obtenida a partir de una encuesta de satisfacción cumplimentada por los estudiantes participantes de forma anónima — siendo 1 la valoración más baja y 5 la más alta—, y que nos muestra su grado de satisfacción con la metodología docente empleada (tabla 3).

Las cifras recogidas en las tablas anteriores arrojan unos valores de la tasa de abandono ligeramente superiores al $25 \%$ de los alumnos que, durante el desarrollo de la actividad, deciden no concluir sus proyectos arquitectónicos; del resto de estudiantes, los que sí completan el cuatrimestre, superaron la asignatura un $87,90 \%$, valor que se traduce en una elevada tasa de éxito; finalmente, un $29,4 \%$ de los alumnos evaluados obtuvieron una calificación superior al estrictamente aprobado - notable o sobresaliente-.

La comparativa de los valores anteriores respecto a los que se aportan a continuación para los cursos académicos siguientes nos permitirá relativizar estas cifras para poder así formular una conclusión que nos permita relacionar la implementación de metodologías de aprendizaje activo basado en proyectos e instrumentos de innovación educativa complementarios con la motivación del alumnado universitario. 


\section{Aprendizaje interdisciplinar en Proyectos Arquitectónicos}

Durante el curso 2017-2018, la asignatura Proyectos 6 incorporaba una nueva variable a la metodología de aprendizaje activo basado en proyectos. En esta ocasión, se formulaba un enunciado en cuyo planteamiento de partida se daba cabida a la interdisciplinaridad. Así, se proponía a los alumnos trabajar sobre un enunciado diseñado por un profesional de una disciplina diferente a la Arquitectura, en este caso, la gestión cultural. Así, tomando como base el programa de actividades de un festival cultural, los alumnos de Arquitectura debían proponer los espacios arquitectónicos que propiciaran el desarrollo de éstas. Bajo el lema "MUCC, Cultura para repensar la ciudad", la actividad involucró a un total de 61 alumnos, a tres docentes de Arquitectura, y a dos gestores culturales externos que participarían al inicio y al final de la experiencia educativa, para marcar las directrices y demandas programáticas de la actividad (figura 1) y para actuar como jurys y valorar de forma objetiva las propuestas finales de los estudiantes (figura 2), respectivamente.

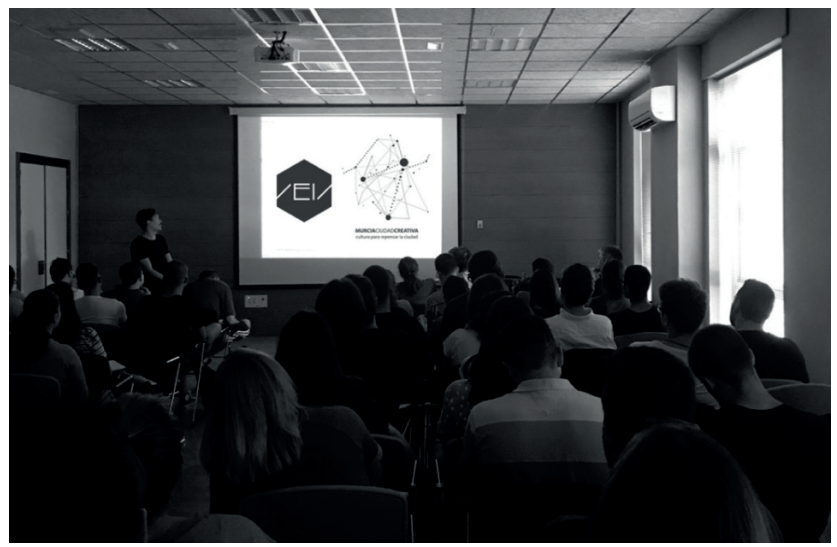

Figura 1. Interdisciplinaridad en Proyectos Arquitectónicos: presentación. Fuente: elaboración propia.

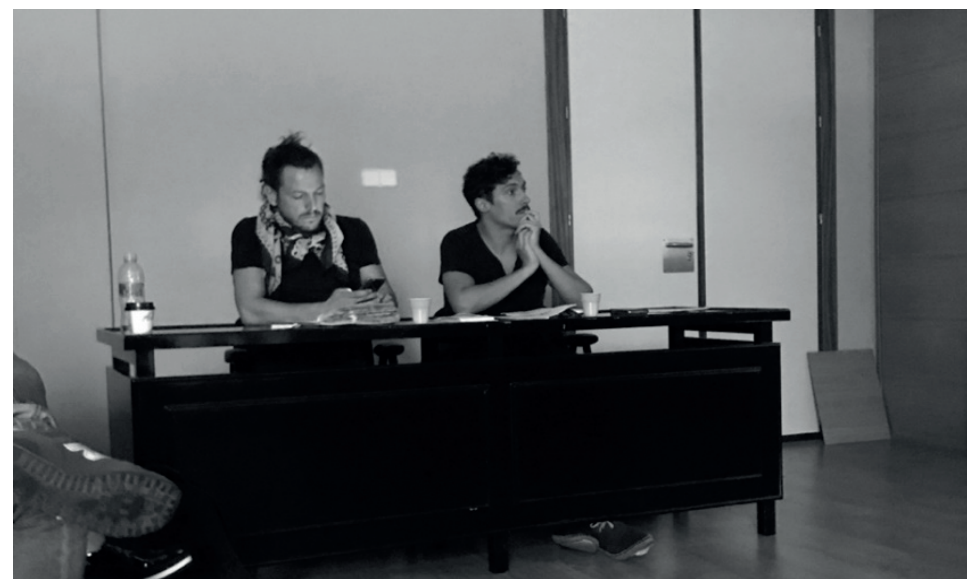

Figura 2. Interdisciplinaridad en Proyectos Arquitectónicos: jury final. Fuente: elaboración propia. 
A la conclusión del curso, se sometió la metodología docente aplicada nuevamente a un doble sistema de valoración: una cuantitativa en los mismos términos que el caso anterior (tabla 4); y una cualitativa que nuevamente recogía los resultados de una encuesta anónima diseñada previamente para someter a examen la metodología docente así como la introducción de la interdisciplinaridad como complemento a ésta (tabla 5).

\begin{tabular}{|l|l|l|l|l|l|l|l|}
\hline \multicolumn{6}{|l|}{ Curso 2017-2018. MUCC, Cultura para repensar la ciudad } \\
\hline NP & Susp. & Ap. & Not. & Sob. & M.H. & Total & \\
\hline 14 & 3 & 33 & 9 & 2 & - & 61 & Tasa de éxito: $93,60 \%$ \\
\hline $\mathbf{2 2 , 9 0 \%}$ & $4,90 \%$ & $54,10 \%$ & $14,80 \%$ & $3,30 \%$ & - & $100 \%$ & Tasa de fracaso: $6,40 \%$ \\
\hline
\end{tabular}

Tabla 4. Resultados cuantitativos aprendizaje interdisciplinar en Proyectos Arquitectónicos. Fuente: elaboración propia.

\begin{tabular}{|l|l|l|l|l|l|l|l|}
\hline \multicolumn{6}{|l|}{ Repercusión de la interdisciplinaridad en la motivación del alumnado universitario } \\
\hline 1 & 2 & 3 & 4 & 5 & Población & Muestra & Media \\
\hline 0 & 1 & 2 & 6 & 6 & 61 & 15 & $\mathbf{4 , 1 3}$ \\
\hline
\end{tabular}

Tabla 5. Resultados cualitativos aprendizaje interdisciplinar en Proyectos Arquitectónicos. Fuente: elaboración propia.

Ante la cuestión que se refería a la interdisciplinaridad y su repercusión sobre su propia motivación e implicación en la asignatura, los estudiantes otorgaban un optimista valor muy por encima del obtenido por otros parámetros también sometidos a evaluación como el trabajo en equipo $(3,67)$, el formato de las entregas $(4,00)$ o la introducción de recompensas o incentivos para los trabajos más notables $(3,60)$.

\section{Aprendizaje-servicio en Proyectos Arquitectónicos}

La última de las metodologías activas basada en proyectos sometida a evaluación ha sido el aprendizaje-servicio (ApS) en Proyectos Arquitectónicos. Si bien esta metodología está ganando cada vez más aceptación en las universidades españolas, algunos autores como Álvarez Castillo, Martínez Usarralde, González González y Buenestado (2017, p. 103) alertan del retraso con el que ésta ha comenzado a implementarse en nuestro país, y de manera más acusada si nos centramos en las enseñanzas universitarias donde su aplicación está resultando "más lenta y costosa que en otros niveles". 
La asignatura Proyectos 6 proponía en el curso 2018-2019 a sus 39 alumnos la participación en una experiencia educativa - “Conexiones Vitales"- donde el aprendizaje no sólo se realizaba de manera activa, sino que todo el conocimiento generado por los estudiantes tenía además una utilidad como prestación de servicios a la Comunidad, traspasando definitivamente todas las fronteras del ente universitario. Y es que, como señalan los autores Romero Ariza y Pérez Ferra (2009, p. 97), la manera en que se diseñan las tareas de aprendizaje tiene también una influencia determinante en la motivación del alumnado universitario. Así:

Cuando el profesor propone una actividad, dicha tarea lleva asociados unos determinados objetivos y demanda un papel concreto por parte del estudiante; por lo tanto, la potencial utilidad de aquello que se ha de aprender - lo que se conoce como el valor de la tarea, muy presente en la metodología de $\mathrm{ApS}$ - es otro de los factores que pueden facilitar la motivación de los estudiantes.

Los alumnos, trabajando de la mano junto con diferentes colectivos y asociaciones vecinales a través de la celebración de talleres colaborativos y participativos — extrapolación de procedimientos de las experiencias speed dating y blind dates, y adaptación a un formato de entrevistas breves y anónimas a los diferentes colectivos participantes-, debían atender las demandas sociales de estos y canalizarlas a través de sus propuestas arquitectónicas con el fin de evidenciarlas frente a la Administración Pública. La participación de la ciudadanía se dio, de nuevo, al inicio del cuatrimestre con la realización de las visitas de trabajo y la realización de los talleres participativos (figura 3), y al final con la celebración de la presentación pública y divulgación de los resultados obtenidos, requisito fundamental de las experiencias de ApS (figuras 4 y 5).

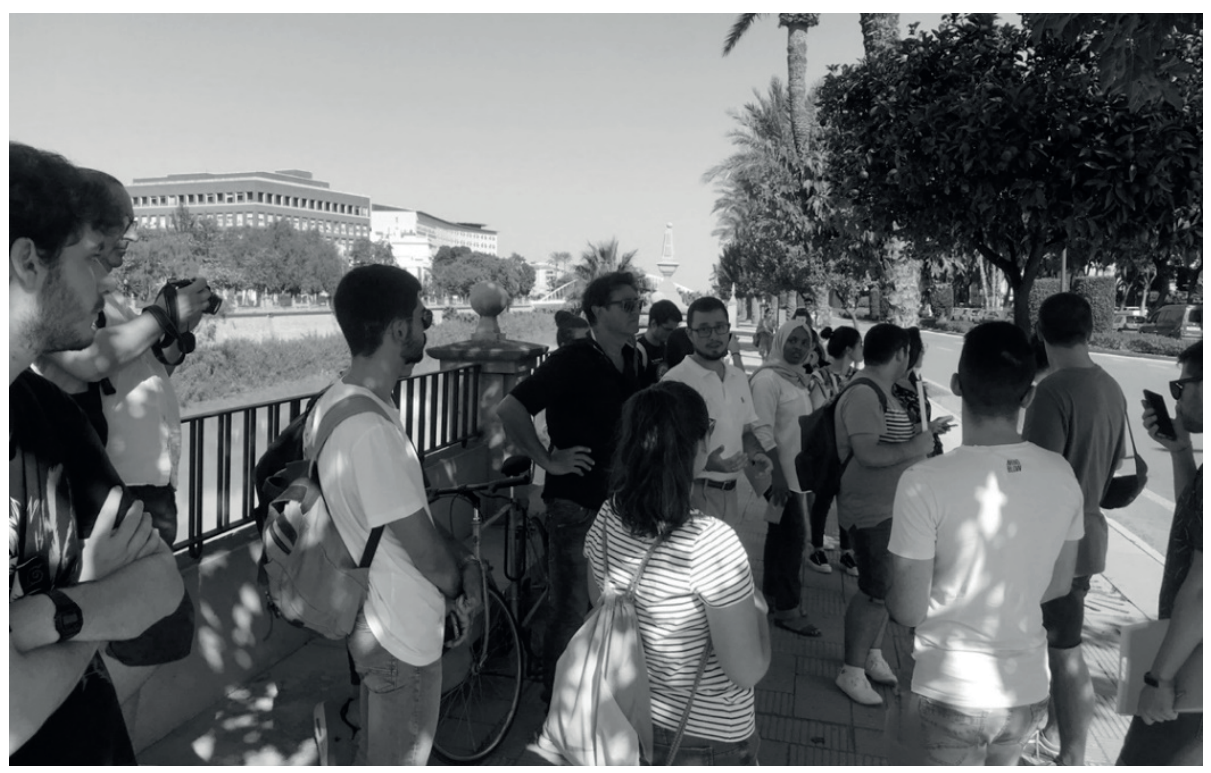

Figura 3. Aprendizaje-servicio en Proyectos Arquitectónicos: visitas de trabajo con los vecinos. Fuente: elaboración propia. 


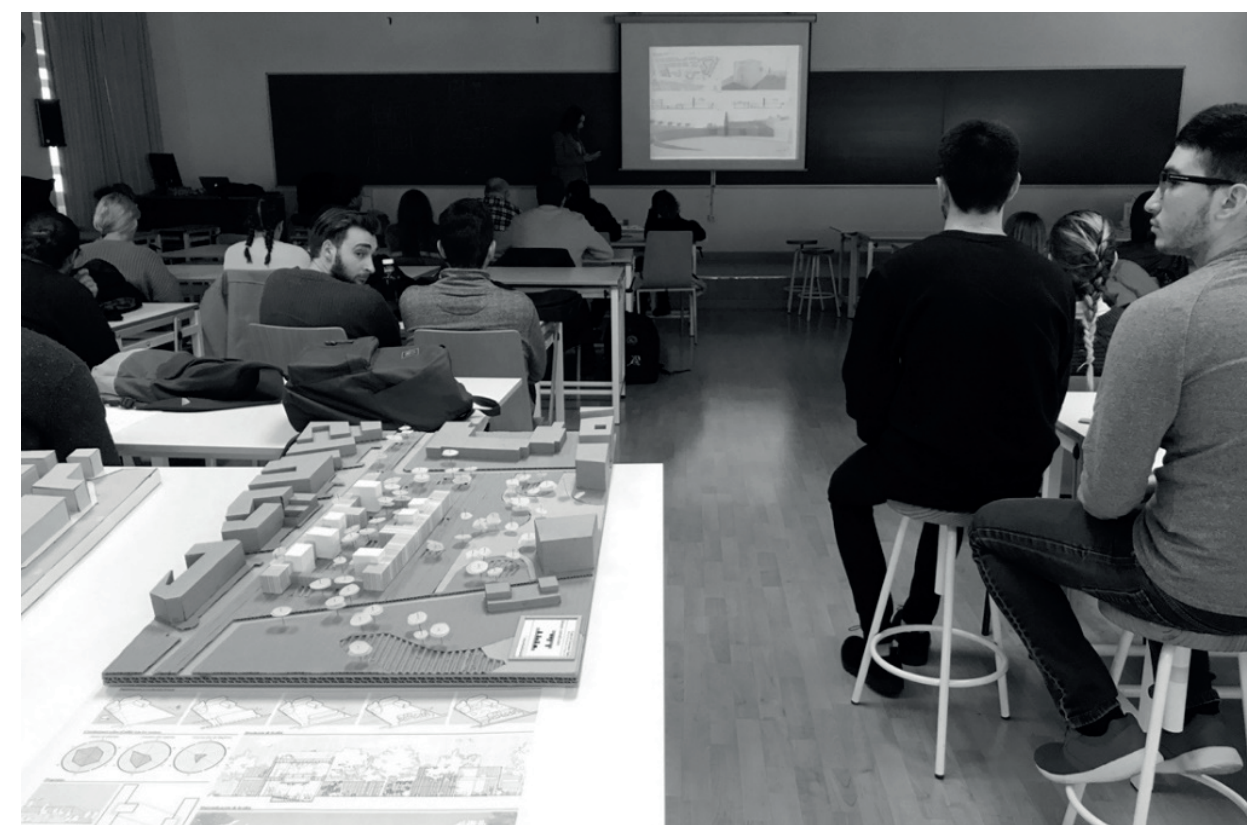

Figura 4. Aprendizaje-servicio en Proyectos Arquitectónicos: presentación y divulgación de resultados. Fuente: elaboración propia.

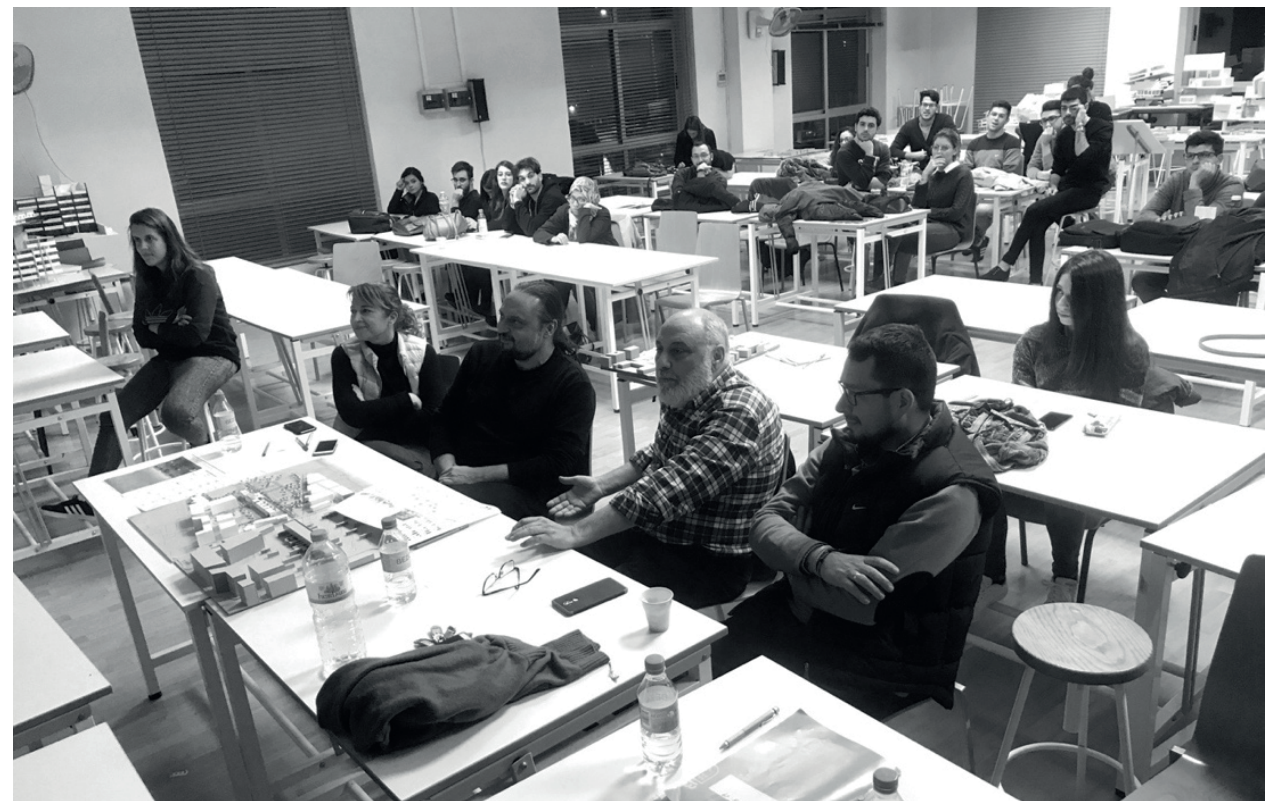

Figura 5. Aprendizaje-servicio en Proyectos Arquitectónicos: presentación y divulgación de resultados. Fuente: elaboración propia. 
Una vez más, y continuando el método establecido para este trabajo, a la finalización del curso se llevó a cabo la evaluación tanto cuantitativa (tabla 6) como cualitativa (tabla 7) de la metodología de ApS, esta última a través de la valoración de la prestación de servicios a la Comunidad como instrumento de motivación para el alumnado universitario — con los mismos instrumentos de recogida de datos y rangos de valoración ya referidos anteriormente-.

\begin{tabular}{|l|l|l|l|l|l|l|l|}
\hline \multicolumn{6}{|l|}{ Curso 2018-2019. Conexiones Vitales } \\
\hline NP & Susp. & Ap. & Not. & Sob. & M.H. & Total & \\
\hline 5 & 0 & 20 & 10 & 4 & - & 39 & Tasa de éxito: $100 \%$ \\
\hline $\mathbf{1 2 , 8 2} \%$ & $0,00 \%$ & $51,28 \%$ & $25,64 \%$ & $10,26 \%$ & - & $100 \%$ & $\begin{array}{l}\text { Tasa de fracaso: } \\
0,00 \%\end{array}$ \\
\hline
\end{tabular}

Tabla 6. Resultados cuantitativos aprendizaje-servicio en Proyectos Arquitectónicos. Fuente: elaboración propia.

\begin{tabular}{|l|l|l|l|l|l|l|l|}
\hline \multicolumn{6}{|l|}{ Repercusión del servicio a la Comunidad en la motivación del alumnado universitario } \\
\hline 1 & 2 & 3 & 4 & 5 & Población & Muestra & Media \\
\hline 0 & 0 & 3 & 10 & 13 & 39 & 26 & $\mathbf{4 , 3 8}$ \\
\hline \\
Valoración global de la metodología de aprendizaje-servicio \\
\hline 1 & 2 & 3 & 4 & 5 & Población & Muestra & Media \\
\hline 0 & 1 & 2 & 4 & 12 & 39 & 19 & $\mathbf{4 , 4 2}$ \\
\hline
\end{tabular}

Tabla 7. Resultados cualitativos aprendizaje-servicio en Proyectos Arquitectónicos. Fuente: elaboración propia.

Con estos valores, se completa la toma de datos y evaluación de las tres diferentes variantes de metodologías de aprendizaje activo basado en proyectos ensayadas para este trabajo, lo que nos permite ahora relacionar cada una de ellas con parámetros como la tasa de abandono, la tasa de éxito, el rango de calificaciones y, finalmente, la propia satisfacción del alumnado participante.

\section{DISCUSIÓNY CONCLUSIONES}

En este trabajo, se ha tratado de fijar un conjunto de parámetros de valoración objetiva que nos permitan evaluar en última instancia el aumento de la motivación del alumno universitario en la asignatura de Proyectos en Arquitectura. A continuación, se muestra un resumen de las evaluaciones realizadas (tabla 8), para posteriormente proceder con la discusión y formulación de conclusiones.

Tasa de abandono. El número de alumnos que durante el desarrollo del cuatrimestre abandonan el curso muestra una progresión a la baja, con un descenso destacable durante el curso 2018-19, lo que nos lleva a presumir una mayor influencia de la metodología activa del ApS sobre la motivación del alumnado respecto de otras variantes sometidas a examen, lo que anima al alumnado a realizar el seguimiento de la asignatura hasta su fase final. 
Tasa de éxito. Si atendemos al grado de superación de la asignatura de todos aquellos estudiantes que participan de la totalidad del curso, la tendencia es en este caso al alza, llegando incluso a alcanzar el valor máximo posible nuevamente en el curso académico donde se implementó la metodología de ApS, en el que la totalidad de los alumnos que completaron el curso obtuvieron calificaciones que les permitín la superación de la asignatura. No obstante, los valores obtenidos en el resto de cursos académicos no resultan tampoco desfavorables, lo que confirma la idoneidad de este tipo de metodologías en las asignaturas de Proyectos en Arquitectura.

\begin{tabular}{|l|l|l|l|}
\hline & $\begin{array}{l}\text { Curso 2016-17 } \\
\text { Aprendizaje } \\
\text { activo }\end{array}$ & $\begin{array}{l}\text { Curso 2017-18 } \\
\text { Aprendizaje } \\
\text { interdisciplinar }\end{array}$ & $\begin{array}{l}\text { Curso 2018-19 } \\
\text { Aprendizaje- } \\
\text { servicio }\end{array}$ \\
\hline Tasa de abandono $(0-100 \%)$ & $26,50 \%$ & $22,90 \%$ & $12,82 \%$ \\
\hline Tasa de éxito $(0-100 \%)$ & $87,90 \%$ & $93,60 \%$ & $100 \%$ \\
\hline $\begin{array}{l}\text { Calificaciones superiores a Aprobado } \\
(0-100 \%)\end{array}$ & $29,40 \%$ & $25,00 \%$ & $35,90 \%$ \\
\hline $\begin{array}{l}\text { Instrumento de innovación educativa } \\
(1-5)\end{array}$ & - & 4,13 & 4,38 \\
\hline Valoración de la metodología (1-5) & 4,17 & 3,90 & 4,42 \\
\hline
\end{tabular}

Tabla 8. Resumen de la evaluación de metodologías docentes. Fuente: elaboración propia.

Rango de las calificaciones. En cuanto al rango de calificaciones obtenidas por los alumnos en los diferentes cursos, también la metodología de ApS propicia que el número de alumnos que obtienen calificaciones superiores al Aprobado estricto — Notable y Sobresaliente— sea más elevado que cuando se emplea una metodología de aprendizaje activo basado en proyectos convencional o incorporando la interdisciplinaridad como instrumento de innovación educativa. Entendemos aquí, por tanto, que este incremento de las calificaciones por encima del Aprobado debe interpretarse como un aumento de la motivación de un alumnado que no se conforma con la mera superación de la asignatura, quizás en parte por la propia responsabilidad que los estudiantes se imponen a sí mismos por el hecho de ofrecer los mejores resultados posibles de sus trabajos académicos a la Comunidad.

Satisfacción del alumnado con los diferentes instrumentos de innovación educativa. Como ya se señaló en puntos anteriores, los instrumentos de innovación educativa implementados como complementos de la metodología de aprendizaje activo basado en proyectos han sido la interdisciplinaridad y la prestación de servicios a la Comunidad - ApS —, habiendo obtenido ambos instrumentos una valoración muy positiva por encima del valor 4 que refuerza el empleo de este tipo de instrumentos pedagógicos para aumentar la motivación de los alumnos universitarios.

\section{Valoración de las metodologías de aprendizaje activo basado en proyectos ensayadas.}

Finalmente, se comparan los resultados de satisfacción del alumnado con cada una de las metodologías docentes empleadas, lo que nos permite establecer cual de ellas ha tenido mayor influencia sobre la motivación de los estudiantes universitarios. En ese sentido, y como ya avanzaban los optimistas resultados de los parámetros anteriores, es la metodología de ApS la que mayor aceptación 
ha tenido entre los alumnos participantes en cada uno de los tres cursos académicos, con una valoración considerablemente superior respecto al resto de variantes que confirma la teoría de Romero Ariza y Pérez Ferra (2009, p. 97) sobre la influencia de la utilidad de la tarea respecto de la motivación de los discentes. Por el contrario, los valores inferiores obtenidos por la metodología de aprendizaje activo basado en proyectos introduciendo la interdisciplinaridad en el planteamiento docente evidencia como la motivación de los estudiantes universitarios disminuye cuando las tareas requeridas salen de su zona habitual de confort, lo que autores como Vicente Oliva y Andrés Tirapo (2007) identifican como "alumnos resistentes al cambio". Éste representa en gran medida uno de los límites que el empleo de estas metodologías activas puede suscitar, si bien la cada vez más creciente promoción que desde las universidades públicas se hace de metodologías como el aprendizaje-servicio hace que éstas tengan cada día más calado en un perfil de estudiante universitario cada vez más comprometido con la prestación de servicio a la Comunidad, como demuestran experiencias recientes como las desarrolladas en la Universidad Politécnica de Cartagena que enmarcan la implementación de este tipo de metodologías en la consecución de los Objetivos de Desarrollo Sostenible — ODS—, y que aquí identificamos como una de las fortalezas de este tipo de metodologías educativas.

\section{NOTAS}

Este trabajo recoge una parte de los resultados de dos Proyectos de Innovación Docente:

1. "Cultura para repensar la ciudad, innovación para repensar la docencia. Aprendizaje activo en Proyectos Arquitectónicos" desarrollado durante el curso académico 2017/2018 en la asignatura Proyectos 6 del Grado en Fundamentos de Arquitectura, al amparo de la convocatoria pública de Proyectos de Innovación Docente 2017-18 según Acuerdo de Consejo de Gobierno de la Universidad Politécnica de Cartagena de 21 de junio de 2017.

2. "Conexiones Vitales: aprendizaje-servicio en Proyectos Arquitectónicos" desarrollado durante el curso académico 2018/2019 en la asignatura Proyectos 6 del Grado en Fundamentos de Arquitectura, al amparo de la convocatoria pública de Proyectos de Innovación Docente 2018-19 según Acuerdo de Consejo de Gobierno de la Universidad Politécnica de Cartagena de 29 de junio de 2018.

\section{REFERENCIAS}

Álvarez Castillo, J. L., Martínez Usarralde, M. J., González González, H. y Buenestado Fernández, M. (2017). El aprendizaje-servicio en la formación del profesorado de las universidades españolas. Revista Española de Pedagogía, 75(267), 199-217. DOI: https://doi.org/10.22550/ REP75-2-2017-02

Carcelén González, R., López Martínez, J. M. y Aroca Vicente, E. (2014). El jury en proyectos arquitectónicos como acción crítica y mecanismo dual de coordinación vertical y transversal en la docencia. Actas II Congreso Internacional de Innovación Docente 2014, Universidad de Murcia. 
Dewey, J. (1916). Democracia y educación. Una introducción a la filosofía de la educación. (Democracy and education. An introduction to the philosophy of education). Madrid: Ediciones Morata.

García Martín, J. y Pérez Martínez, J. E. (2018). Aprendizaje basado en proyectos: método para el diseño de actividades. Tecnología, Ciencia y Educación: revista de carácter científico multidisciplinar, (10), 37-63.

Graaff, E. de y Kolmos, A. (2003). Characteristics of problem-based learning. International Journal of Engineering Education, 19(5), 657-662.

Huber, G. L. (2008). Aprendizaje activo y metodologías educativas. Revista de Educación, número extraordinario 2008, 59-81.

Keller, J. M. (1987). Development and use of the ARCS model of instructional design. Journal of Instructional Development, 10(2). DOI: https://doi.org/10.1007/BF02905780

Rinaudo, M. C., Chiecher, A. y Donolo, D. (2003). Motivación y uso de estrategias en estudiantes universitarios. Su evaluación a partir del Motivated Strategies Learning Questionnaire. Anales de psicología, 19(1), 107-119.

Romero Ariza, M. y Pérez Ferra, M. (2009). Cómo motivar a aprender en la Universidad: una estrategia fundamental contra el fracaso académico en los nuevos modelos educativos. Revista Iberoamericana de Educación, (51), 87-105.

Santander, G. (2017). Aprendizaje servicio como herramienta metodológica en la educación superior. RIDAS, Revista Iberoamericana de Aprendizaje Servicio, (3), 159-163. DOI: https://doi. org/10.1344/RIDAS2017.3.13

Silberman, M. (1998). Aprendizaje activo: 101 estrategias para enseñar cualquier tema. (Active Learning: 101 strategies for teach any subject). Buenos Aires, Argentina: Editorial Troquel.

Valero, E. (2006). Ocio peligroso: introducción al proyecto de arquitectura. Valencia: General de Ediciones de Arquitectura.

Vicente Oliva, S. y Andrés Tirapo, A. (2007). Resistencia de los alumnos al aprendizaje activo. Actas I Jornadas Innovación Docente, Tecnologías de la Información y la Comunicación e Investigación Educativa en la Universidad de Zaragoza: caminando hacia Europa. Universidad de Zaragoza. 